\title{
Generation of phospholipid vesicle-nanotube networks and transport of molecules therein
}

\author{
Aldo Jesorka ${ }^{1}$, Natalia Stepanyants ${ }^{1}$, Haijiang Zhang ${ }^{1}$, Bahanur Ortmen ${ }^{2}$, Bodil Hakonen ${ }^{1} \&$ Owe Orwar $^{1}$
}

${ }^{1}$ Department of Chemical and Biological Engineering, Chalmers University of Technology, Göteborg, Sweden. ${ }^{2}$ Department of Molecular Biology \& Genetics, Bilkent University, Ankara, Turkey. Correspondence should be addressed to O.O. (orwar@chalmers.se).

Published online 19 May 2011; doi:10.1038/nprot.2011.321

\begin{abstract}
We describe micromanipulation and microinjection procedures for the fabrication of soft-matter networks consisting of lipid bilayer nanotubes and surface-immobilized vesicles. These biomimetic membrane systems feature unique structural flexibility and expandability and, unlike solid-state microfluidic and nanofluidic devices prepared by top-down fabrication, they allow network designs with dynamic control over individual containers and interconnecting conduits. The fabrication is founded on self-assembly of phospholipid molecules, followed by micromanipulation operations, such as membrane electroporation and microinjection, to effect shape transformations of the membrane and create a series of interconnected compartments. Size and geometry of the network can be chosen according to its desired function. Membrane composition is controlled mainly during the self-assembly step, whereas the interior contents of individual containers is defined through a sequence of microneedle injections. Networks cannot be fabricated with other currently available methods of giant unilamellar vesicle preparation (large unilamellar vesicle fusion or electroformation). Described in detail are also three transport modes, which are suitable for moving water-soluble or membranebound small molecules, polymers, DNA, proteins and nanoparticles within the networks. The fabrication protocol requires $\sim 90$ min, provided all necessary preparations are made in advance. The transport studies require an additional 60-120 min, depending on the transport regime.
\end{abstract}

\section{INTRODUCTION}

Biological cells have developed a complex, truly nanoscale infrastructure for transport, biochemical synthesis, information processing, sensing and other life-sustaining molecular activities ${ }^{1-3}$. Nanotubular structures are quite common in biological systems, in particular for establishing cell-to-cell connections $s^{4}$. Intercellular nanotubes (ICNs) formed from filopodial structures or after separation of tight cell-cell contacts mediate transport processes between cells. For example, diffusion of inositol triphosphate through nanotubes possibly mediates calcium signaling between macrophages, a gradient of membrane tension can induce transport of membrane patches and surface proteins, and molecular motors can transport organelles along filamentous actin in $\mathrm{ICNs}^{5-7}$. Land plants have developed plasmodesmata, a form of sophisticated membrane channel used to span the rigid cell walls to mediate the cell-to-cell exchange of signaling molecules ${ }^{8}$.

It remains a challenging engineering task to model, design and build artificial devices that precisely probe, interface, imitate or even augment chemical or physical key features of biological systems ${ }^{9-13}$. Nanotube-vesicle networks (NVNs) are versatile, man-made soft-matter constructs of micrometer and nanometer dimensions that can, in this context, serve as models for interlinked biochemical reactors ${ }^{14,15}$, which are closely related to naturally occurring ICN nanotube assemblies ${ }^{3}$. The networks are fabricated from individual phospholipid molecules by a combination of selforganization processes, which lead to spherical bilayer membrane compartments, and subsequent forced shape transition operations, which transform selected membrane regions into nanotubes and new liposomes. The networks are useful as spatially separated, yet interconnected, chemical reactors of differentiated contents, allowing the study of membrane proteins and (bio)chemical reactions on biologically relevant size scales ${ }^{16,17}$.
The protocol described here is schematically illustrated in Figure 1. It combines a preparation procedure for giant unilamellar vesicles (GUVs) connected to multilamellar membrane reservoirs with electroporation and microinjection methods for the extraction of lipid nanotubes, the growth of new vesicles and their controlled surface adhesion. To create, investigate and apply vesicle-nanotube networks for transport and chemical reactions, these methods have been unified and integrated with a variety of microscopy setups, such as differential interference contrast, wide-field laser-induced fluorescence and confocal microscopy ${ }^{15}$.

The network fabrication is based on generation of GUVs from phospholipids. An important requirement is the coexistence of unilamellar and multilamellar vesicles (MLVs), as the process of 'daughter' vesicle creation requires a continuous supply of membrane material. Few of the numerous liposome preparation methods ${ }^{18}$ are appropriate for this purpose. A modified procedure by Criado and Keller $^{19}$, starting from a purified lipid extract of soybean material, has proven to be the most suitable ${ }^{20}$. This dehydration/rehydration procedure involves the deposition of a lipid film by evaporation of an organic solvent in a rotating flask; this is followed by brief ultrasonication and swelling of the resulting lipid film in phosphate buffer (Fig. 1, stage I). The liposome preparation is then dehydrated in vacuo, and subsequently rehydrated to form GUVs with attached multilamellar reservoirs (Fig. 1, stage II).

These soft-material structures, when immobilized on thin transparent slides, can be subjected to investigation and manipulation under a microscope ${ }^{20}$. Giant unilamellar phospholipid vesicles are tens of micrometers in diameter and harbor picoliters to femtoliters of internal volume ${ }^{21,22}$. The soft character of the lipid bilayer allows penetration with microneedles after membrane pore opening by electric fields (Fig. 1, stage III). Microinjection with glass capillaries 
Figure 1 | Schematic overview over the network generation procedure. The procedure consists of five fundamental fabrication subprocedures and three subsequently possible transport experiments. The pink thread in the background of the scheme depicts the sequential order of the operations. Building of larger or topologically more advanced networks, depicted as an optional sub-procedure using dashed pink lines, involves repeated daughter vesicle creation and deposition. The images are illustrated as simplified, not-to-scale side views. Black triangles depict translation micromovements of needle or electrode, and red arrows symbolize the transport direction of network-internalized entities. Positive injection pressure is denoted by $+\mathrm{P}$; electrode polarity is also indicated for the electroporation and field-driven transport operations. The procedure detailed in this protocol starts at stage II (de/rehydration) and uses two-vesicle assemblies as elementary network structures for transport experiments.

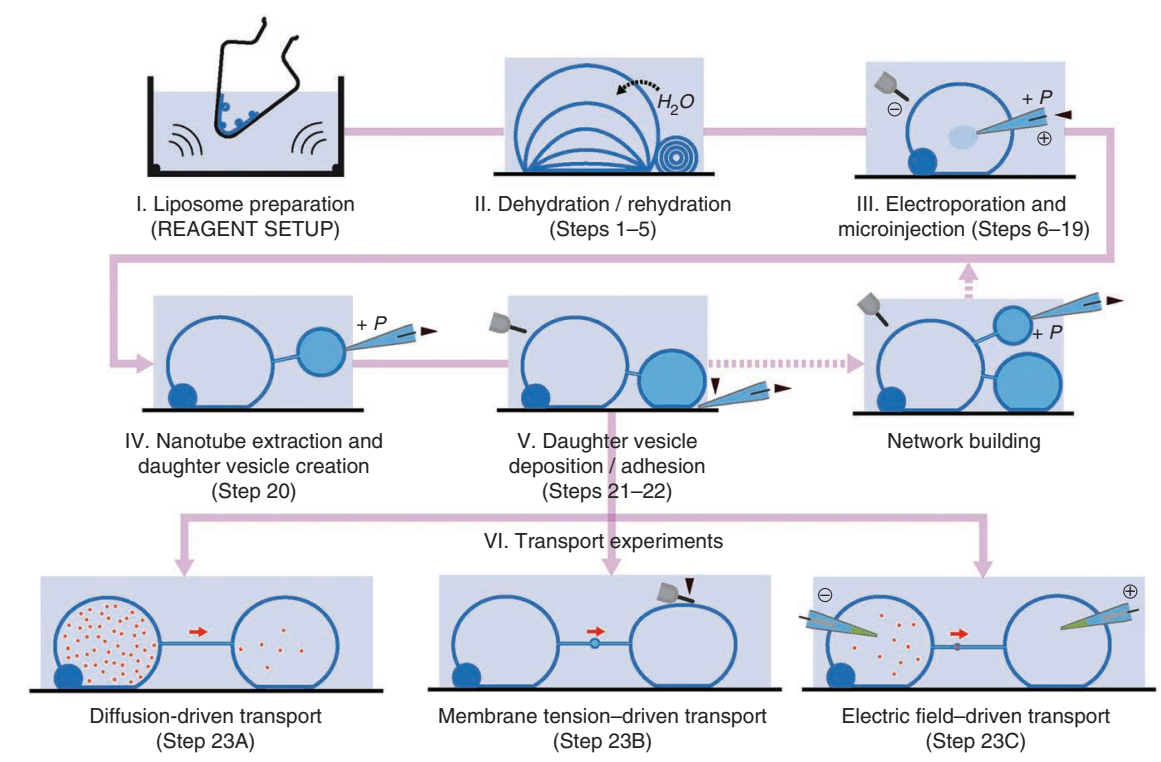

and the associated micromanipulation methods date back to almost a century ${ }^{23}$, and basic micropipette setups have remained almost unchanged in principle because of the sturdiness and simplicity of the technique ${ }^{24}$. Highly repeatable fabrication procedures and tools for microneedles became widely available just after the patchclamp method for single-cell electrophysiology was developed ${ }^{25}$. A capillary pulling instrument is crucial for the generation of NVNs. Similarly, the electroporation method, originally established for direct delivery of genetic material ${ }^{26}$ or chemical compounds ${ }^{27,28}$ into the interior of biological cells, has central importance in network fabrication, as stable pores allow facile introduction of microneedles with quite large orifices into bilayer containers (electroinjection) $)^{20,29}$. Once a needle is inside a GUV, the bilayer tightly seals around the tip. The adhesion of the membrane is typically strong enough to allow the pulling of lipid material from the membrane, thereby instantly forming an open nanoconduit between the vesicle and the capillary. The flexible lipid nanotubes created in this way can be expanded at the needle-connected end by means of positive injection pressure, to an extent that a new vesicle is formed (Fig. 1, stage IV). Constant subsequent injection of fluid from the needle allows the 'daughter' vesicle to grow to a size appropriate for deposition onto the substrate (Fig. 1, stage V). Iteration of this pulling and vesiclegeneration process enables the building of whole networks of interconnected containers, in which size, geometry and individual contents can be controlled. If a new needle with different internal solution is used for the generation of each new vesicle, a network with differentiated contents can be created. The composition of the internal volume of each newly created vesicle is determined by the size ratio of mother and daughter vesicles ${ }^{30}$.

As the nanoconduits are open on both ends and thus truly interconnect the individual containers, exchange of internal solution, and chemical compounds dissolved therein, is possible. Different regimes of exchange of matter have been investigated by us. In particular, the transport of small molecules and nanoparticles by diffusion ${ }^{31-33}$, by membrane tension-driven (Marangoni) flow $^{34}$ and through electrophoretic migration ${ }^{35,36}$ were studied in detail (Fig. 1, stage VI).

Although diffusion and electrophoresis are rather common transport processes often encountered in the biosciences, the transport of material through nanotubes by tension-driven (Marangoni) flow of the lipid membrane is a noteworthy specialty that has been investigated in greater detail ${ }^{37}$. A flow of lipids is created along the tube membrane from the region of low membrane tension to a region of high membrane tension, which is temporarily created by pressing a vesicle with a carbon fiber. Material transport occurs through a plug flow induced in the tube by the flowing membrane. The membrane of an NVN is in the low-tension regime ${ }^{38}$, in which tension increases exponentially with area $\operatorname{strain}^{39}$. However, as the applied tension gradients are small, a series expansion around the initial tension gives a linear relation between tension and strain ${ }^{37}$.

Internalization of fluorescent compounds or labeled chemical entities in network-integrated vesicles as well as staining of the membranes by lipophilic fluorescent probes allow application of modern microscopy techniques to investigate a variety of aspects, such as membrane and transport dynamics ${ }^{40}$, biocatalysis ${ }^{31,41}$ and nanotube properties ${ }^{38,42}$. The biomimetic nature of the network has also opened pathways to the study of membrane proteins ${ }^{17}$ and the association of biological cells to networks ${ }^{43,44}$.

\section{Objectives of this technique}

Here we describe a thoroughly tested protocol for generating NVNs from commonly available materials and sources ${ }^{14,20}$, as well as for performing three different network internal transport operations $^{31,32,34,35,37}$. Because of their micro- and nanoscale size and flexibility, networks are highly suitable for the construction of biomimetic chemical reactor models and for the study of the transport and interaction of chemical and biochemical reagents under conditions of close confinement. Models for intracellular reaction chains and biochemical kinetics, molecular crowding, functional aspects of artificial cells, ion channels and other membrane proteins are among the topics that can become more accessible to scientists, while allowing a high degree of integration with existing microscopy and micromanipulation systems.

\section{Previous techniques and alternative methods}

As NVNs are, in essence, combinations of nanofluidic devices ${ }^{45}$ and microreactors ${ }^{46}$, solid-state microfluidic and nanofluidic devices accommodate some of their functions and properties ${ }^{47-49}$. Cell blebbing ${ }^{50}$ is an alternative method by which to obtain unilamellar 
membrane compartments with certain, albeit limited, functionality; it is useful, in particular, for the study of membrane proteins in their native environment ${ }^{17}$. Moreover, innovative biomimetic techniques for the study of membrane-related processes and biomolecular interactions associated with membranes have been developed ${ }^{51,52}$. Few of these techniques can provide the biocompatibility, structural flexibility and expandability that the individually designed soft-matter network infrastructures provide. A variety of alternative methods exist for the preparation of phospholipid vesicles of the type used in this protocol. MLVs can be conveniently prepared by a simple and robust film-hydration technique ${ }^{53}$ or by spray drying/lyophilization ${ }^{54}$ for larger-scale preparations. There is no reliable method to produce exclusively GUVs; the electroformation method reaches the highest yields ${ }^{55,56}$, especially when used in combination with spin coating of a lipid film ${ }^{57}$. Electroformation is limited to low ionic strength buffers, but methods to increase the ion strength have been developed ${ }^{58}$.

There are also alternative methods to produce lipid nanotubes. Examples include streptavidin binding to biotinylated membranes ${ }^{59}$, pulling action of molecular motors ${ }^{60}$, and optical tweezers $^{61}$. The tubes are generated, either by shape-transforming phospholipid vesicles or by applying point forces to vesicles or biological cells, but such protrusions are typically not connected to other vesicles or cells. Moreover, the electroformation procedure has been found to spontaneously produce nanotubes between GUVs $^{62,63}$. We are not aware of any equivalent method to create soft-matter networks from lipid membranes with controlled and individual design. Solid-state microfluidic and nanofluidic devices do not have the flexibility, dimensions or biomimetic properties of vesicle-nanotube networks.

\section{Applications and limitations}

Vesicle-nanotube networks are uniquely shapeable and have been functionalized and tailored to provide insights into microscale phenomena, such as local confinement of reactants and catalysts to initiate and follow the progress of enzymatic reactions ${ }^{16,31}$. Membrane protein integration was demonstrated ${ }^{64}$, as was the interconnection and interaction with biological cells and active surfaces ${ }^{17,43,44}$. Vesicle content control and differentiation ${ }^{30}$, as well as external control of the outside solution composition, provide a large variety of possible chemical environments, and dynamic sub-compartmentalization has opened pathways to more advanced artificial cell models ${ }^{65}$.

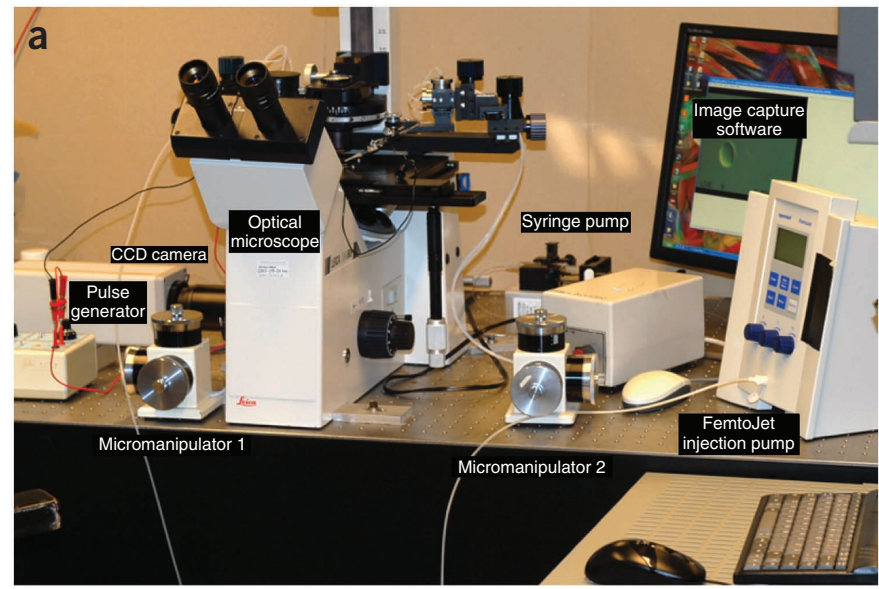

Single-molecule transport and spectroscopy have been reported ${ }^{66}$, and fundamental properties and the dynamics of lipid nanotubes $^{38,42,67}$, which have an important role in intercellular transport and communication ${ }^{5}$, have been investigated.

Limitations come partly from the properties of the soft-matter materials used to build these networks and partly from restrictions on experimental conditions. Lipids are, for example, biomaterials prone to oxidative damage on prolonged exposure to air $^{68}$, and the experimental setup, essentially a 'lab in a droplet', suffers from evaporation loss and gradually increasing osmotic stress on the networks. Furthermore, it is not possible to reproducibly fabricate identical networks, nanotube lengths and vesicle diameters, and vesicle positions on the surface vary. However, the use of patterned surfaces in combination with specific adhesion strategies can be used to define vesicle locations more accurately ${ }^{69}$. Automated procedures for network fabrication do not exist yet.

Introduction of chemical materials into networks is rather easily achieved, but removal of products or unwanted components from the network is more challenging and has thus far barely been studied. Other limitations arise from the simple internal structure of vesicles and their networks, which is not comparable with the complex internal machinery of biological cells. Active flow control within networks, which is very desirable, has been studied to some extent; however, open questions remain with respect to valve designs, function and stability ${ }^{70}$.

\section{Experimental design}

The network generation protocol is essentially a micromanipulation and microinjection procedure that relies on techniques common in cell electrophysiology, in vitro fertilization and microscopy. The flexibility of the soft-matter networks allow a broad variety of different experimental techniques to be applied to the study of the desired feature. Liposome preparation from soybean lipid extracts requires a minimum of laboratory hardware, including a rotary evaporator, ultrasonic bath and glassware. Chemicals and materials are largely inexpensive and available from common sources. Attention has to be directed to acquisition of proper micromanipulation hardware, in particular, under the perspective of successful integration into an existing microscopy system. Figure 2 shows our accordingly modified wide-field laser-induced fluorescence microscopy setup. In addition to the components of

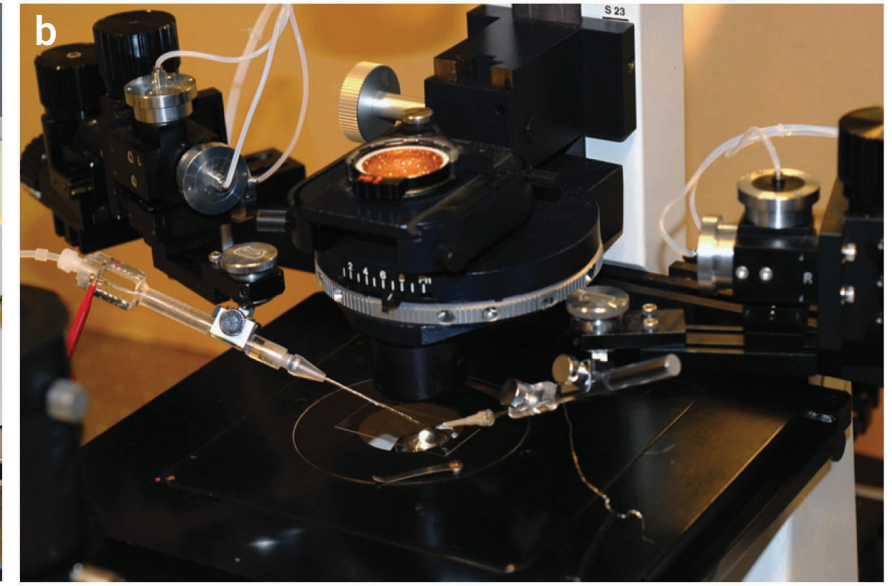

Figure 2 | Optical differential interference contrast microscopy setup for nanotube-vesicle network fabrication. (a) Required components assembled on an optical table. (b) Detail of the microscope table, showing the arrangement of the coarse micromanipulator pair with mounted capillary needle/needle holder and carbon fiber microelectrode/microelectrode holder, as well as their electrical connector clamps. 
Figure 3 | Capillary needle and carbon fiber microelectrode preparation. (a) Sutter Instruments P-2000 UV-laser capillary puller with a mounted needle, programmed for long-tapered needles with a $250 \mathrm{~nm}$ opening. The parameters for this one-line program are as follows: Heat 430, FIL 4, VEL 50, DEL 240 and PUL 100. (b) Carbon fiber microelectrode (top) and the components needed to assemble it: plastic-encapsulated $5-\mu \mathrm{m}$ (OD) carbon fiber, 5-cm-long (OD, $1 \mathrm{~mm}$ ) glass capillary, 0.25-mm (OD) silver wire and two-component conductive silver epoxy. (c) Capillary (top) and microelectrode holder (bottom). The capillary holder body with FemtoJet tube-compatible thread is in-house built to fit the 8-mm micromanipulator mount. A fine-threaded screw at the rear end has a silver wire soldered to it, which runs inside the holder along the long axis and reaches into the capillary needle. The holder tip is a commercially available patch pipette grip head (Eppendorf). The microelectrode holder is from Narishige.

this setup, most importantly microscope, micromanipulators, pulse generator and injection pump, proper capillary fabrication and filling equipment is required, including a puller, a flame forger and means of backfilling pipette tips. Each commercial capillary puller has its own set of parameters to achieve a particular needle profile, taper and orifice diameter. Most manufacturers provide instructions. Figure 3a shows our laser puller, displaying the simple oneline program that we created to obtain needles for the procedure described here. When creating new needle programs, we recommend confirming the resulting shape and dimensions, for example, by means of a scanning electron microscope.

Microelectrodes for electroporation and electrophoresis are easily fabricated from raw wires or semi-manufactured commercial components such as plastic-encapsulated carbon fibers. The injection needle holder, with its internal electrode and removable head, is an in-house development designed to fit the holding clamps of our particular micromanipulator model. All individual components are shown in Figures 3 and 4.

Surface coating of the substrate slides is recommended to ensure network stability over prolonged time periods. The preparation of coated (SU-8 photoresist) microscope cover slips is not strictly required, but provides the most suitable surfaces, which ensure reliable repeatability of the experiments. Ordinary borosilicate or soda lime glass cover slips will give similar results, but suffer decreased longevity of the networks produced thereon. Coating the glass

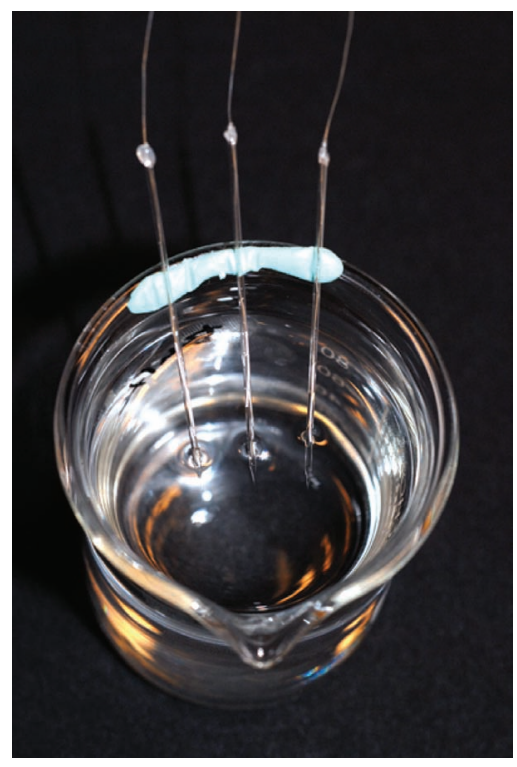

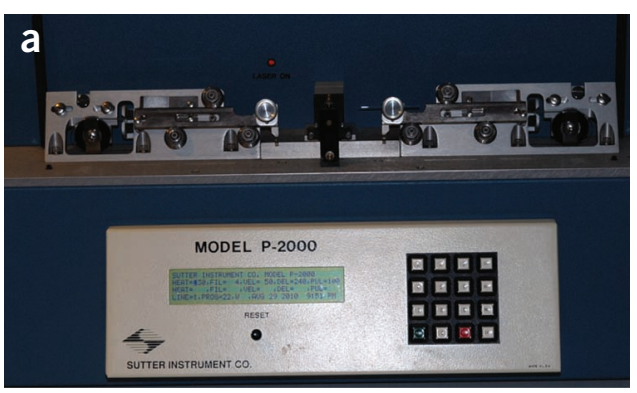
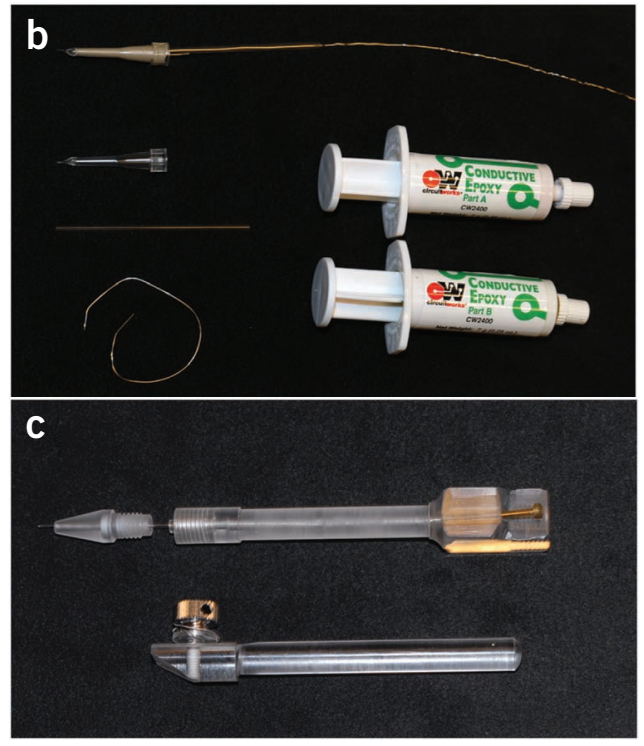

cover slips with a thin $(\sim 5 \mathrm{~nm})$ gold film is an alternative, which also helps to promote rapid adhesion of vesicles to the substrate. Gold-coated slides suffer from decreased optical transparency, and fluorescence quenching can occur close to the surface.

A clean-room facility with a spin coater and mask aligner is optimal, but effective SU-8 coating of microscope cover slips can be achieved under standard laboratory conditions with the help of a 2,000 r.p.m. bench-top spin coater, a 365-nm UV lamp and a hot plate.

Necessary experimental considerations for the microscopy part of the protocol are the type of microscope needed, e.g., bright-field differential interference contrast (DIC), confocal or fluorescence, the objective used and the imaging and data processing options. We use $\times 40$ or $\times 63$ objectives (Leica, HCX PL APO, $\times 40 / \times 63$, NA $1.25 / 1.32$, oil immersed) for our confocal and wide-field laserinduced fluorescence microscopes. Both systems are placed on airdampened optical tables, an essential measure to avoid vibration of the microneedles, and both feature optional manual or motorized translation tables. Available light sources/wavelengths define the possible selection of fluorescent labels to be used to visualize the networks. Reagents and dyes must be chosen accordingly. Image acquisition requires a color digital camera, as well as conveniently accessible and sufficiently powerful software to record and analyze its output.

Figure 4 | Electrophoresis electrode assemblies, each consisting of a 1-mm (OD) hydrophobized capillary needle with cross-linked polyacrylamide gel plug and internal $\mathrm{Ag} / \mathrm{AgCl}$ electrode. The capillaries are sealed with silicone rubber and are stored immersed in electrolyte. 
Fine micromanipulators are best attached directly to the microscope, but can also be mounted onto the dampening table, e.g., by means of a magnetic base. The latter option tends to increase the effect of needle vibrations, greatly influenced by the dimensions of the base and height of the microscope stage above the table.

Depending on the size and complexity of the network to be fabricated with this procedure and the nature of study to be performed with it, the time required for a full experiment can be a few hours. In this case, droplet evaporation loss needs to be taken into consideration and the droplet be replenished with distilled water, either manually in intervals or in an automated fashion, with the help of a pump supplying pure water at a constant rate (typically approximately $50-100 \mu \mathrm{l}$ every $30 \mathrm{~min}$ ) through a thin polytetrafluoroethylene tube. The actual loss is best determined before an experiment, using a balance.

The protocol described here has been thoroughly tested only on soybean polar extract (SPE), but not on pure single lipids or entirely artificial lipid mixtures. If networks are to be prepared from those precursors, the initial lipid preparation step must be modified to yield appropriate mixtures of unilamellar and MLVs, which can then be used in network building. We have found that, when pure lipids are used instead of SPE, the addition of $\sim 0.1 \%$ cholesterol is essential to obtain stable MLVs. Hydration/dehydration is still the only method available to obtain suitable mixtures of multilamellar and giant unilamellar liposomes, and we have not yet established a network fabrication procedure for pure lipids. Factors affecting the yield of formation are the degree of bilayer separation, (influenced by temperature and glycerol content), the lipid composition and the ionic composition of the surrounding buffer. Negatively charged lipids such as phosphatidyl serines or ethanolamines are known to enhance separation of the lamellae. Relatively low ionic strength buffer (10-50 $\mathrm{mM})$ and the absence of multivalent ions that interlink charged lipids can also be beneficial.

Desired lipophilic membrane constituents such as glycolipids, fluorescently tagged lipids, biotinylated or antibody-modified lipids, membrane proteins, small hydrophobic sensor molecules and polymers can be added as a minor fraction, typically below $5 \mathrm{~mol} \%$, to the liposome pre-preparation, without compromising the ability to form GUVs and networks ${ }^{69}$.

Biological cells have been used as lipid source for direct network generation, using the same order of steps as in the protocol described above, to maintain the natural membrane composition and preserve the orientation of embedded membrane proteins ${ }^{17}$. The injection of more viscous, gel-forming polymer solutions into networks for the purpose of flow control and subcompartmentalization has also been reported ${ }^{65,70,71}$. The network preparation procedure remains essentially the same, although it requires wider needle openings and microfabricated functional substrates.

\section{MATERIALS}

\section{REAGENTS}

$\triangle$ CRITICAL All reagent solutions and distilled water must be filtered through $0.2-\mu \mathrm{m}$ membrane filters $(0.45 \mu \mathrm{m}$ for nanoparticle suspensions) before use in the experiments. Reagents are American Chemical Society grade or of higher purity.

\section{Lipid preparation}

- Soybean polar (lipid) extract (SPE; Avanti Polar Lipids,

product no. 541602C) $\Delta$ CRITICAL Use of other lipids than SPE requires adjustments of the preparation step.

- Glycerol (Sigma, product no. G5516)

- Chloroform (Sigma, product no. 650498)

Phosphate buffer solution

- Potassium phosphate $\left(98 \% ; \mathrm{K}_{3} \mathrm{PO}_{4}\right.$, Sigma, product no. P5629) ! CAUTION It is irritating to skin and also poses the risk of serious damage to eyes.

- Potassium dihydrogen phosphate $\left(\mathrm{KH}_{2} \mathrm{PO}_{4}\right.$, VWR, product no. 26936.236)

- Trizma (Sigma, product no. T1503)

- EDTA (Fluka, product no. 03609)

- Magnesium sulfate $\left(\mathrm{MgSO}_{4} \cdot 7 \mathrm{H}_{2} \mathrm{O}\right.$, Merck, product no. 1.05886)

- Phosphoric acid $\left(\mathrm{H}_{3} \mathrm{PO}_{4}\right.$, Sigma, product no. P5811)! CAUTION It causes burns. It also poses the risk of serious damage to eyes. $\triangle$ CRITICAL The PBS buffer cannot easily be exchanged by other buffer systems, such as HEPES, as phosphate ions were found to be important to promote self-assembly of the lipid vesicles during rehydration.

\section{Polyacrylamide hydrogel}

- Acrylamide (98\%; Fluka, product no. 01700; 99\%; Sigma, product no. A3553) ! CAUTION It is toxic and possibly teratogenic. It is readily absorbed through skin. Wear appropriate safety gloves. Latex gloves do not offer sufficient protection.

- $N, N^{\prime}$-methylenebisacrylamide (98\%; Fluka, product no. 66670; 99\%; Fluka, product no. 66667)! CAUTION It is hazardous.

- Ammonium persulfate ((NH4) $\mathrm{S}_{2} \mathrm{O}_{8}$, Aldrich, product no. 431532)

! CAUTION It is an oxidizer. It may cause allergic skin reactions.

- $N, N, N^{\prime}, N^{\prime}$-tetramethylethylenediamine (TEMED; Sigma,

product no. T9281) ! CAUTION It is caustic and has an irritating smell; handle only under a fume hood.

Dye label solutions for injections and membrane staining

- Atto 488 (water-soluble acridine dye $\left(\lambda_{\text {exc }}=488 \mathrm{~nm}\right.$ ); Atto-Tec, product no. AD488-21) $\Delta$ CRITICAL Atto 488 gives the best photobleaching performance, compared with cyan-green-fluorescent dyes, such as Fluorescein, Alexa Fluor 488 or Dylight 488.

- DiD oil ( $1,1^{\prime}$-dioctadecyl-3,3,3',3'-tetramethylindodicarbocyanine perchlorate, membrane-soluble carbocyanine dye $\left(\lambda_{\mathrm{exc}}=644 \mathrm{~nm}\right)$; Invitrogen, product no. D307)

- Carboxylated latex beads $\left(20 \mathrm{~nm}\right.$, yellow/green FluoSpheres, $\lambda_{\text {exc }}=505 \mathrm{~nm}$; Invitrogen, product no. F8787) ! CAUTION Fluorescent dyes can lead to various short- and long-term skin photosensitization and cell-damaging effects. Avoid contact.

Surface coating

- Negative photoresist SU-8 2002 (Microchem, product no. Y111029)

! CAUTION It contains cyclopentanone as solvent and is highly flammable.

Electrophoresis electrode preparation

- Hexamethyldisilazane (HMDS; Sigma, product no. 379212)! CAUTION It is highly flammable and is harmful if inhaled, if it comes in contact with skin and if swallowed. It also causes burns.

- Hexanes (Sigma, product no. 139386) ! CAUTION It is highly flammable and is harmful if swallowed. It is irritating to eyes, respiratory system and skin.

- Potassium chloride (KCl, Sigma, product no. P9333)

- Silver wire (OD (outer diameter) $0.25 \mathrm{~mm}$; Goodfellow, product no. AG005140)

- Gold or platinum wire (OD 0.25 mm; Goodfellow, product no. AU005140 or PT005140)

\section{REAGENT SETUP}

PBS buffer preparation $\bigcirc$ TIMING 15 min Dissolve $0.151 \mathrm{~g}$ Trizma base, $1.592 \mathrm{~g} \mathrm{~K}_{3} \mathrm{PO}_{4}, 1.021 \mathrm{~g} \mathrm{KH}_{2} \mathrm{PO}_{4}, 0.062 \mathrm{~g} \mathrm{MgSO}_{4} \cdot 7 \mathrm{H}_{2} \mathrm{O}$ and $0.0465 \mathrm{~g}$ EDTA in $250 \mathrm{ml}$ distilled water. Adjust the $\mathrm{pH}$ with $\mathrm{H}_{3} \mathrm{PO}_{4}$ to 7.8 . Store at $4{ }^{\circ} \mathrm{C}$ for a maximum of 1 month, but warm to room temperature $\left(\sim 21^{\circ} \mathrm{C}\right)$ before use. Liposome preparation $\bigcirc$ TIMING $18 \mathrm{~h}$ (once, lasts for multiple experiments) Liposomes are created following a procedure by Cirado and Keller $^{19}$, which yields GUVs attached to multilamellar membrane reservoirs. The procedure is optimized for SPE, a natural plant lipid mixture. Artificially 
created mixtures or individual lipids need modifications, in particular cholesterol addition, to give a comparable result.

Prepare a $100 \mathrm{mg} \mathrm{ml}^{-1}$ lipid stock solution by dissolving $100 \mathrm{mg}$ SPE in $1 \mathrm{ml}$ of chloroform. Transfer a $30-\mu \mathrm{l}$ aliquot of the stock solution to a $10-\mathrm{ml}$ round bottom flask and add $300 \mu \mathrm{l}$ chloroform. Evaporate on a rotary evaporator at 6-8 $\mathrm{kPa}$ vacuum and $80-100$ r.p.m. for $5 \mathrm{~h}$ to remove the solvent and form a lipid film. Add $3 \mathrm{ml}$ of buffer and allow the lipid film to rehydrate for $12 \mathrm{~h}$ at $4{ }^{\circ} \mathrm{C}$ in a refrigerator. Add $30 \mu \mathrm{lglycerol}$ to the suspension and sonicate for 5-10 min in an ultrasonic bath. Partition $150-\mu \mathrm{l}$ aliquots into 20 Eppendorf tubes $(0.5 \mathrm{ml})$ and store at $-18^{\circ} \mathrm{C}$ for a maximum of 6 months. $\triangle$ CRITICAL All manipulations of lipid solutions should be performed using thoroughly cleaned glassware. Do not use flasks, containers, syringes or pipettes made from plastics. $\Delta$ CRITICAL If it is desired to fluorescently label the whole liposome preparation, for example, to visualize the liposome membrane in every experiment, add 1-2 $\mu \mathrm{l}$ DiD oil directly into the lipid solution in chloroform. Otherwise, prepare membrane staining stock solution to use in individual experiments for membrane labeling.

Membrane staining dye solution and nanoparticle suspension preparation - TIMING 30 min First, dissolve $1 \mathrm{mg}$ of $\mathrm{DiD}$ (oil) membrane staining dye in $1 \mathrm{ml}$ fresh, anhydrous DMSO to obtain a membrane staining stock solution. Store dye stock solutions at $-18^{\circ} \mathrm{C}$ under light exclusion for a maximum of 6 months. Second, dilute $25 \mu \mathrm{l}$ of fluorescent carboxylated latex nanosphere suspension (FluoSpheres; yellow/green fluorescent latex beads, diameter $20 \mathrm{~nm}$ ) in $1 \mathrm{ml}$ buffer. Pass this particle injection suspension slowly through a $0.45-\mu \mathrm{m}$ membrane syringe filter. This injection suspension is ready for backfilling and can be stored for up to 1 month at $2{ }^{\circ} \mathrm{C}$ under light exclusion, before particle aggregation interferes. Instead of nanoparticle suspensions, water-soluble fluorescent dye solutions can be injected into liposomes. A larger variety of absorption wavelengths is available for fluorescent dyes than for nanospheres. To prepare a dye solution with absorption/emission properties comparable with the FluoSpheres, dissolve $1 \mathrm{mg}$ of water-soluble fluorescent dye, such as Atto $488\left(\lambda_{\text {exc }}=488 \mathrm{~nm}, M=804 \mathrm{~g} \mathrm{~mol}^{-1}, \varepsilon_{\max }=\right.$ $\left.90,000 \mathrm{M}^{-1} \mathrm{~cm}^{-1}\right)$, in $1 \mathrm{ml}$ buffer to obtain an injection dye stock solution. Pass the injection dye stock solution slowly through a $0.2-\mu \mathrm{m}$ membrane syringe filter. The solution can be stored for several months at $-18^{\circ} \mathrm{C}$ under light exclusion.

\section{EQUIPMENT}

\section{Lipid preparation}

- Rotary evaporator (Büchi R-144)

- Vacuum desiccator

- Ultrasonic bath

\section{Glass pipette preparation}

- Capillary borosilicate glass tubes (OD $1 \mathrm{~mm}$, ID (inner diameter) $0.78 \mathrm{~mm}$, with filament; Harvard apparatus, product no. 300038)

- Capillary puller (Sutter Instrument, model no. P-2000)

- Heat filament forger (Narishige, MF-900)

Surface fabrication

- Microscope cover slips no. 1 (borosilicate glass, $25 \mathrm{~mm} \times 50 \mathrm{~mm}, 0.15 \mathrm{~mm}$ thickness; VWR, cat. no. 631-0146)

- Spin coater (2,000-3,000 r.p.m.; Laurell WS-400-6NPP-LITE/IND spinner, Laurell)

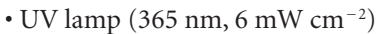

- Hot plate

Carbon fiber electrodes

- Glass cutter

- Plastic-encapsulated carbon fibers (OD $5 \mu \mathrm{m}$; Dagan, ProCFE)

- Electrically conductive epoxy (ELFA, product no. 80-867-12)

- Silicone rubber

Microscopy

- Optical microscope setup with DIC optics (Leica DM IRB, Leica)

$\Delta$ CRITICAL The arrangement of the micromanipulation and microscopy components into a complete experimental setup is shown in Figure 2a.

Figure $\mathbf{2} \mathbf{b}$ is the detail of the microscope table that shows the positioning of the injection needle in its holder and the counter electrode toward each other.

-Wide-field illumination setup with laser (Spectra-Physics 2017) or

fluorescence lamp

- Filter/dichroic mirror set for $\lambda_{\text {exc }}=488 / \lambda_{\text {em }}=525 \mathrm{~nm}$ (ref. 66)
- An objective with $\times 40$ or $\times 63$ magnification $(\times 40 / \times 63$, NA 1.25/1.32, oil immersed; HCX PL APO, Leica); $\times 40$ magnification is optimal for most experiments

- Confocal microscope (Leica DM IRE2 with TCS SP2 scanner, Leica) with laser excitation sources for $\lambda_{\text {exc }}=488 / 495 \mathrm{~nm}$ and $633 \mathrm{~nm}$

Micromanipulation and microinjection

- Two water-hydraulic micromanipulators (coarse/fine; Narishige MC-35A/ MHW-3, Narishige)

- Polycarbonate capillary holder with internal electrode and patch pipette grip head (Eppendorf, cat. no. 920007414)

- Two polycarbonate electrode holders (Narishige H-7, Narishige)

- Rectangular pulse generator (0-99 V), with adjustable pulse length and width between 1 and 2,000 ms (Digitimer DS2A, Digitimer)

- Femtoliter automatic injection pump (Eppendorf FemtoJet, Eppendorf)Automatic micropipette (20-200 $\mu \mathrm{l})$ with Eppendorf microloader tips (Eppendorf, order no. 5242 956.003)

- Disposable syringes ( $5 \mathrm{ml}$; VWR, product no. 613-3951)PVDF membrane syringe filters $(0.2 \mu \mathrm{m}$; VWR, product no. 514-4014)

- Polytetrafluoroethylene filters ( $0.45 \mu \mathrm{m}$; VWR, product no. 514-4003) ! CAUTION To keep the membrane soft, membrane syringe filters often contain small amounts of poly(ethylene glycol), which is gradually being washed out when the syringe filter is used. The network fabrication protocol is not known to be influenced by this additive. Membrane filters should be washed with distilled water before use, if contamination is expected to be an issue.

Optional

- Syringe pump with plastic tubing, with ID $0.4 \mathrm{~mm}$, for replenishment of evaporated buffer (Syringe pump NE-500 OEM)

Electromigration and electrode fabrication

- Power supply (0-6 V; ELFA Agilent U8002A, Agilent)

Image analysis and processing

- Color CCD camera (Hamamatsu C5810, Hamamatsu) $\Delta$ CRITICAL If multiple fluorescent labels are used simultaneously, for example, for the membrane and the vesicle interior, a digital color camera is recommended. If only one fluorescent label is needed, a monochrome camera can be used, such as the Chameleon CMLN-13S2M USB 2.0 camera (Point Grey Research).

- Image recorder (Sony DSR-11 data tape recorder with Firewire interface for direct image transfer to a personal computer, Sony)

- Capture, editing and analysis software (VirtualDub and Image-J)

\section{EQUIPMENT SETUP}

Cover slip surface treatment $O$ TIMING $60 \mathrm{~min}$ for eight pieces Surface treatment is beneficial to fine-tune the adhesion of vesicles to the surface. Bisphenol A-based amplified epoxy resists such as Microchem SU-8 are only moderately hydrophilic, thus preventing strong adhesion to the surface and extending the lifetime of the networks.

Place and immobilize a new cover slip on the spin coater. Pipette $1 \mathrm{ml} \mathrm{SU}-8$ 2002 onto the cover slip. Spin at 2,000-3,000 r.p.m. for 60 s. Dry at $95{ }^{\circ} \mathrm{C}$ on a hot plate for $5 \mathrm{~min}$. Expose with UV light for $15 \mathrm{~s}$ at $6 \mathrm{~mW} \mathrm{~cm}^{-2}$. After exposure, bake at $95^{\circ} \mathrm{C}$ on a hot plate for $5 \mathrm{~min}$. Store in an airtight container away from light.

Capillary needle preparation $\bigcirc$ TIMING 15 min for ten pieces Blow the interior of a OD 1.0/ID $0.78 \mathrm{~mm}$ borosilicate tube with a dry nitrogen stream to remove dust and glass particles. Round both ends of the sharp tube in a capillary forger. This procedure has no influence on the capillary properties, but is strongly recommended to prevent rapid wear of the capillary holder pressure seal. Mount the capillary on the capillary puller and set the instrument to produce long-tapered needles with an opening of approximately $0.2-0.5 \mu \mathrm{m}$. Pull the needle. Figure 3a shows a suitably programmed UV-laser capillary puller with a mounted needle (after pulling), using a one-line program to achieve a needle orifice of $\sim 250 \mathrm{~nm}$. Store the needles with their pointed end slightly elevated in a dust-free container. $\Delta$ CRITICAL Different capillary pullers produce needles of slightly different shapes and tip diameters. Initial establishment of an individual needle program is required. Using 'Heat 430, FIL 4, VEL 50, DEL 240 and PUL 100 ' as needle program, our UV-laser capillary puller produces long-tapered needles with orifices of $\sim 250 \mathrm{~nm}$.

Carbon fiber electrode fabrication $\bigcirc$ TIMING $60 \mathrm{~min}$, including epoxy hardening Cut a $1.0 \mathrm{~mm}(\mathrm{OD})$ borosilicate glass tube to a length of $5 \mathrm{~cm}$ with a glass cutter or scribe. Insert a $10-\mathrm{cm}$-long silver wire such that a 
1-cm-long end protrudes from the tube. Mix the conductive silver epoxy and apply a drop of $2-3 \mathrm{~mm}$ in diameter to the protruding end. Insert the glass rod with the epoxy-covered end quickly into the plastic-covered carbon fiber, such that the epoxy fills the plastic holder completely. Apply more epoxy glue if necessary. Store upright with the carbon fiber pointing downward for $1 \mathrm{~h}$ before use, to make sure the silver epoxy has hardened sufficiently. Figure $3 \mathbf{b}$ shows the components and an assembled electrode. Several carbon fibers should be fabricated at the same time. They can be stored for several months when vacuum-sealed to prevent oxidation of the silver wire. Electrophoretic transport pipette preparation O TIMING $65 \mathrm{~min}$ Dissolve $160 \mathrm{mg}$ HMDS in $100 \mathrm{ml}$ dry hexane. Immerse a capillary tube in this solution for $10 \mathrm{~min}$, rinse with hexane and blow dry with nitrogen. Pull and forge a capillary as described above, using the HMDS-treated capillaries. Prepare a solution of $200 \mathrm{mg}$ acrylamide and $10 \mathrm{mg} N, N^{\prime}$-methylenebisacrylamide in $1 \mathrm{ml}$ water (stock solution A). Prepare a solution of $100 \mathrm{mg}$ (NH4) ${ }_{2} \mathrm{~S}_{2} \mathrm{O}_{8}$ in $1 \mathrm{ml}$ of water (stock solution B). The stock solutions can be stored at $4{ }^{\circ} \mathrm{C}$ for up to 6 months. Place the stock solutions in a water/ice bath for 5 min. Mix $250 \mu \mathrm{l}$ stock solution A with $10 \mu \mathrm{l}$ stock solution B. Add $1 \mu \mathrm{l}$ $N, N, N^{\prime}, N^{\prime}$-tetramethylethylenediamine and mix rapidly for $1 \mathrm{~s}$ to obtain the pre-gel solution. Keep this pre-gel solution in the water/ice bath to slow gel formation. Rapidly take up $25 \mu \mathrm{l}$ pre-gel solution into an Eppendorf microloader tip with an automatic pipette. Rapidly insert the long, narrow microloader tip into the wide capillary needle end and backfill with the pre-gel solution, avoiding bubbles. Bubbles formed occasionally in the needle tip can be displaced by repeated pumping action of the automatic pipette. Dispense only as much pre-gel solution as is necessary to fill the tapered section of the capillary ( $\sim 2 \mathrm{~mm})$. This filling procedure has to be done rapidly, as after $\sim 30 \mathrm{~s}$ gel formation sets in. Allow 10 min to fully polymerize the pre-gel solution. Dissolve $750 \mathrm{mg} \mathrm{KCl}$ in $100 \mathrm{ml}$ water and backfill the gel-capped capillary needle to $\sim 2 \mathrm{~cm}$ length. Pour $50 \mathrm{ml} \mathrm{KCl}$ solution into a beaker, and dip both the silver and the gold (or platinum) wire $1 \mathrm{~cm}$ deep into the $\mathrm{KCl}$ solution. Connect the positive pole of a $6 \mathrm{~V} \mathrm{DC}$ power supply to the silver, and the negative pole to the gold wire. Electrolytic formation of silver chloride sets in. Continue the electrolysis until a thin white layer is formed on the wire ( $\sim 15 \mathrm{~s})$. Insert the wire ( $\mathrm{Ag} / \mathrm{AgCl}$ electrode) into the backfilled capillary. Close the end and fix the wire with a drop of silicon glue. Create at least one more electrode by this procedure and store the electrophoresis electrodes with their tips submerged in the $\mathrm{KCl}$ solution (Fig. 4). $\triangle$ CRITICAL Backfilling of the capillary must be performed without delay. The pre-gel solution solidifies within $30 \mathrm{~s}$ after addition of the amine component. $\triangle$ CRITICAL Other gel-forming materials such as agarose can replace the acrylamide/bisacrylamide system.

\section{PROCEDURE}

\section{De-/rehydration of lipid $\bigcirc$ TIMING $30 \mathrm{~min}$}

1| Warm a frozen liposome preparation (see REAGENT SETUP) to room temperature.

2| Pipette $5 \mu$ l of the lipid suspension onto an untreated glass cover slip.

3| Place the cover slip in a vacuum desiccator and evaporate the solution at $<100 \mathrm{kPa}$ vacuum (membrane pump) for 10-30 min. The dehydrated lipid forms a beveled transparent film with salt crystals in the center (Fig. 5a, right side). Glycerol prevents complete dehydration.

4| Pipette $0.5 \mathrm{ml}$ PBS buffer onto the dehydrated lipid spot and rehydrate for 5 min to swell into giant vesicles (Fig. 5a, left side). ? TROUBLESHOOTING

5| Transfer the rehydrated vesicle suspension to the SU-8-coated (see EQUIPMENT SETUP; Fig. 5b, right side) or uncoated (Fig. 5 b, left side) cover slip and mix with $0.5-1 \mathrm{~mL}$ PBS buffer. Figure 6 shows typical vesicle suspensions on a glass cover slip before (Fig. 6 a) and after (Fig. 6b) transfer to a new cover slip.

\section{Network generation $\bigcirc$ TIMING $60 \mathrm{~min}$}

6| Switch to differential interference contrast imaging on the microscope.

7| Place the cover slip with the transferred vesicle suspension on the microscope.

8| Attach a needle holder (Fig. 3c, top) and an electrode holder (Fig. 3c, bottom) to the micromanipulator (see Fig. 2b).

9| Take up 25-100 $\mu$ L PBS buffer into an Eppendorf microloader tip using an automatic pipette.

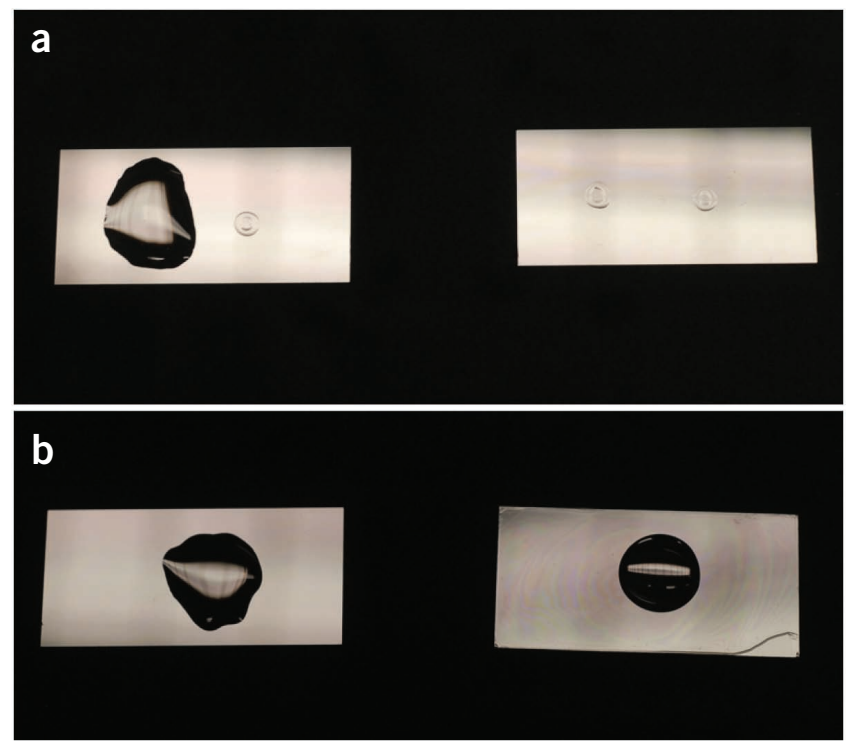

Figure 5 | Lipid dehydration/hydration on no. 1 microscope cover slips and subsequent transfer. (a) Cover slips with two 5 - $\mu$ l lipid suspension droplets after desiccation (right) and rehydration (left). The lipid droplets dehydrate to form beveled spots with buffer salt crystals in the center. (b) After rehydration, the droplets are transferred to SU-8 surface-modified (right) or unmodified (left) borosilicate glass cover slips. 
Figure 6 | Rehydration/swelling of lipid preparations. (a) DIC micrograph of swelling lipids after addition of buffer to a dehydrated lipid droplet. Large numbers of vesicles of different sizes at different stages of swelling and a mostly lipid-covered surface are visible. (b) After transfer of the buffer containing the swollen liposomes to a new cover slip, a greatly reduced number of GUVs is immobilized on a clean substrate surface.
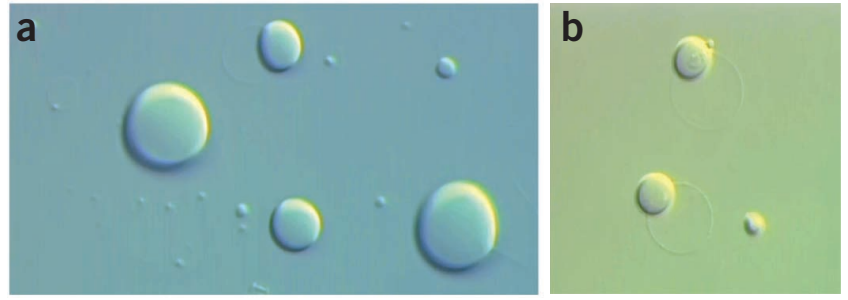

10| Insert the long, narrow microloader tip into the back end of a capillary needle (produced as described in the EQUIPMENT SETUP) and backfill with $15 \mu$ of the buffer; avoid bubble formation.

$\triangle$ CRITICAL STEP Air bubbles trapped in the tip will interfere with the microinjection and electroporation. Use only filamented glass capillaries, as the filament guides the liquid into the tip. Bubbles formed occasionally in the needle tip can be easily displaced by repeated pumping action of the automatic pipette.

11| Insert the backfilled capillary needle into the capillary holder. Fasten the holder head so that the capillary is held pressure-tight.

12| Attach the supply tube of a FemtoJet injection pump to the end of the capillary holder. Adjust the compensation pressure to 2-3 $\mathrm{hPa}$.

13| Attach a carbon fiber microelectrode (produced as described in the EQUIPMENT SETUP) to the electrode holder.

14| Connect the positive pole of a pulse generator to the capillary holder center contact, and the negative pole to the silver wire of the carbon fiber microelectrode.

15| Use the coarse micromanipulators on the stage (see Fig. 2b) to bring microelectrode and needle close to the center of the cover slip, above the objective aperture.

16| Focus the microscope on the surface and locate the vesicles.

17| Refocus the microscope $\sim 100 \mu \mathrm{m}$ above the surface and then manipulate both needle and microelectrode into focus, using first the coarse and then the fine micromanipulators. Slowly descend with the focus to the surface, adjusting the needle height accordingly with the fine micromanipulators.

\section{? TROUBLESHOOTING}

18| Select a vesicle and manipulate microelectrode and needle to opposite sides of the vesicle (Fig. 7a).

\section{? TROUBLESHOOTING}

19| Bring the needle in contact with the vesicle, reduce the compensation pressure to $0 \mathrm{hPa}$ and apply a pulse of $20 \mathrm{~V}$, $10 \mathrm{~ms}$ duration to the membrane. Brief motion of the membrane near the needle is noticeable. Manipulate the needle into the vesicle using the fine micromanipulators (Fig. $\mathbf{7 b}$ ). If the needle does not penetrate the membrane, repeat the pulse and then increase the voltage by increments of $5 \mathrm{~V}$, until the membrane responds. Depending on the size of the needle orifice, the necessary electroporation voltage varies.

$\triangle$ CRITICAL STEP Proper pulse parameters and coordination between the needle translation, pulsing and simultaneous reduction of compensation pressure is important to introduce the needle. Injection pulse voltages over $30 \mathrm{~V}$ and of durations over $100 \mathrm{~ms}$ should be avoided. They result in rapid decay of the carbon fiber microelectrode.
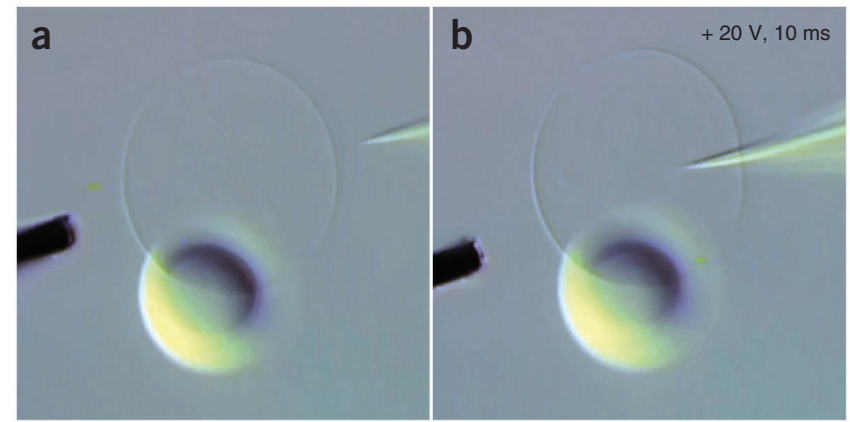
? TROUBLESHOOTING

Figure 7 | Initial stage of network fabrication. (a) A GUV with attached multilamellar lipid reservoir is arranged between a $5-\mu \mathrm{m}$ carbon fiber microelectrode and an injection capillary needle. Both needle and electrode are positioned by water-hydraulic micromanipulators. (b) Application of an electroporation pulse (+20 V, $10 \mathrm{~ms}$ ) with simultaneous translation at $0 \mathrm{hPa}$ backflow compensation pressure inserts the needle into the vesicle. The membrane closes around the needle and self-seals instantly. 
Figure 8 | Stepwise fabrication of a linear network from a single GUV/MLV. (a) After penetration of the membrane, a continuous injection pressure of $10-15 \mathrm{hPa}$ is set and the needle is retracted with $\sim 5 \mathrm{~m} \mathrm{~s}^{-1}$ linear velocity as indicated by the red arrow. (b) A daughter vesicle is forming at the tip of the needle, constantly growing in size. (c) The vesicle is adhered to the surface and the process is repeated to generate further containers, if desired, with a needle of different content to generate diverse internal volumes.

20| Increase the compensation pressure to $10-15 \mathrm{hPa}$, and slowly retract the needle with $\sim 5 \mu \mathrm{m} \mathrm{s}^{-1}$ linear velocity

(Fig. 8a). As soon as the needle leaves the vesicle, a daughter vesicle is formed at the tip, constantly growing in size.
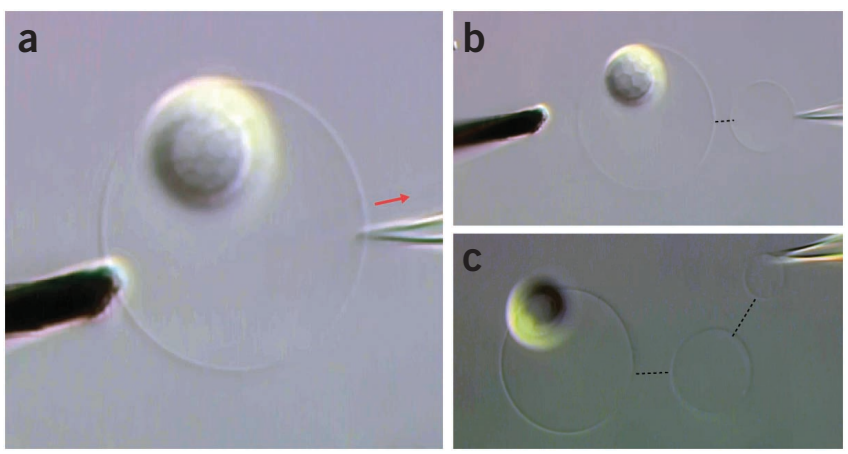

$\Delta$ CRITICAL STEP Position the vesicle close by the mother, to ensure further growth, and increase the compensation pressure to $30 \mathrm{hPa}$. The greater the distance, the slower the new vesicle will increase in size, even at high injection pressure.

? TROUBLESHOOTING

21 When a desired size is reached, reduce the compensation pressure to 1-2 $\mathrm{hPa}$ and manipulate the vesicle to a position to adhere it to the surface (Fig. 8 b).

22 Adjust the microscope focus close to the surface ( 10 $\mu \mathrm{m}$ down) so that the needle touches the surface lightly. Pause several minutes to let the vesicle adhere tightly. Reverse polarity at the pulse generator. Slowly retract the needle and apply a pulse of $20 \mathrm{~V}$, for $10 \mathrm{~ms}$ duration, to release the vesicle. Manipulate the needle up, and switch back to normal pulse polarity. A two-vesicle network has been created, which is the foundation for each of the three following transport experiments.

$\triangle$ CRITICAL STEP Repeat the procedure either at the mother or the new daughter vesicle to create further daughter vesicles. If the procedure is repeated at the daughter vesicle, a linear network ensues (Fig. 8c), if at the mother vesicle, a branched network forms (Fig. 9a,b). Figure 9c shows an example of a branched network on the first daughter vesicle, which is created either directly from the daughter vesicle or, alternatively, by translating a vesicle along an existing nanotube ${ }^{16,31}$. Once-used needles can be reused for further injections, as long as sufficient backfilled solution remains and the tip is not damaged or contaminated. If a high membrane tension regime for the network is desired, the multilamellar reservoir can be cleaved from the network by means of the carbon fiber microelectrode.

\section{? TROUBLESHOOTING}

\section{Transport experiments within networks}

23| Transport experiments within networks can be undertaken using option A for diffusion-driven transport, option B for Marangoni transport or option C for electrophoretic transport:

\section{(A) Diffusion-driven transport in networks TIMING 140 min}

(i) Add $10 \mu \mathrm{l}$ membrane staining dye solution in DMSO to the PBS buffer droplet containing the vesicle network from Step 22 using an automatic micropipette. Wait 5 min for the dye to migrate into the vesicle membranes (Fig. 10a,b).

$\Delta$ CRITICAL STEP This transport mode is most conveniently carried out using confocal microscopy imaging, allowing two different excitation wavelengths for membrane dye and injected nanoparticles. The dye amount in this step is adequate for confocal microscopy. The observed fluorescence intensity depends on excitation light intensity and camera sensitivity. Check the fluorescence intensity and add another aliquot if desired. If DiD (oil) membrane staining dye was added to the initial liposome preparation (see REAGENT SETUP), there is no need to add it here.

(ii) Pull a new injection capillary and backfill it with $15 \mu \mathrm{l}$ of filtered fluorescent nanoparticle suspension (0.02\% solid content, see REAGENT SETUP).
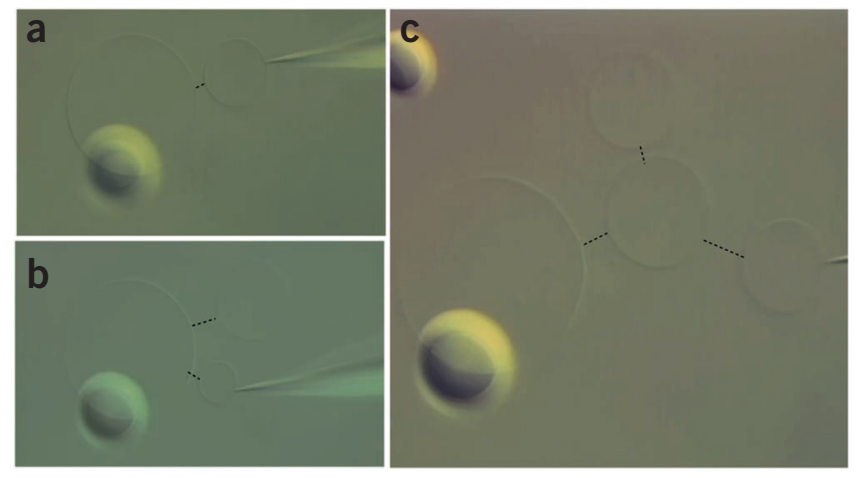

(iii) Remove the needle slowly and carefully from the two-vesicle network on the cover slip surface using the fine micromanipulator, followed by the coarse micromanipulator.

Figure 9 | Stepwise fabrication of a branched network from a single GUV/MLV. (a-c) After generation of the first daughter vesicle (a), a second vesicle can be generated from the mother vesicle (b) and positioned adjacent to the first daughter vesicle (c). The flexible nanotube migrates along the existing tube in a zipper-like fashion ${ }^{38}$ between the two already surface-adhered vesicles, leading to a tube between the two daughter vesicles. Additional vesicles can thus be created and connected to selected containers. 
Figure 10 | Confocal microscopy images showing diffusive transport of internalized 20-nm fluorescent latex nanoparticles between container vesicles through a nanotube. The membrane is stained with DiD, a lipophilic carbocyanine dye (magenta), and the internalized hydrophilic nanoparticles are modified with a fluorescent rhodamine dye (cyan). The excitation wavelength $\lambda_{\text {exc }}=633 \mathrm{~nm}$ for DiD (oil),

for the internalized nanosphere particles it is $495 \mathrm{~nm}$ and the emission bands were set to 640-690 nm and 505-545 nm, respectively. (a) Bright-field (transmission) image of the mother vesicle before network generation. (b) Confocal fluorescence micrograph of the two-vesicle network; the membrane fluorescence channel is selectively displayed. (c) Confocal fluorescence micrograph of the two-vesicle network immediately after nanoparticle injection (0 min). The nanoparticle fluorescence channel is selectively displayed. (d) Confocal fluorescence micrograph of the same fluorescence channel after $120 \mathrm{~min}$, showing the migrated particles in the daughter container and the nanotube. The detector gain has been increased in comparison to $\mathbf{c}$, to better visualize the fluorescence from the nanotube.

(iv) Remove the needle from the droplet using the coarse micromanipulators. Inspect the network and ensure that both vesicles are firmly attached to the surface.

(v) Replace the injection needle with the one backfilled with fluorescent nanoparticle suspension from Step 23A(ii) and move it to the surface, close to the mother vesicle.

\section{? TROUBLESHOOTING}

(vi) Inject the nanoparticle suspension into the mother vesicle for $4-5 \mathrm{~s}$ at $30 \mathrm{hPa}$ injection pressure. Figure 10c shows a confocal microscopy image of a two-vesicle network immediately after injection of fluorescent nanoparticles $(t=0 \mathrm{~min})$ into the mother vesicle.

\section{? TROUBLESHOOTING}

(vii) Retract the needle carefully and move it out of the droplet with the fine and then the coarse micromanipulators.

(viii) Wait for $60 \mathrm{~min}$, with the excitation source blocked or turned off. Occasionally, check the fluorescence from the daughter vesicle to ensure vesicle integrity. Figure $10 \mathrm{~d}$ shows a confocal microscopy image at $t=120 \mathrm{~min}$, showing fluorescence from the nanoparticles in the daughter vesicle.

$\triangle$ CRITICAL STEP Other fluorescent dyes or nanoparticles are appropriate, depending on the available excitation wavelengths. If stained membranes are to be used on a regular basis, membrane-soluble fluorescent dye can be added to the initial vesicle preparation. Confocal microscopy is the most suitable technique for diffusion-driven transport experiments ${ }^{31}$, but wide-field laser fluorescence microscopy is also appropriate.

\section{? TROUBLESHOOTING}

\section{(B) Marangoni transport in networks $\bigcirc$ TIMING 60 min}

(i) Inspect the orientation of the newly created two-vesicle network from Step 22 to ensure that the daughter vesicle can be reached by both the carbon fiber and the needle without affecting the mother vesicle. If necessary, readjust the angle of fiber and/or needle toward the network such that only the daughter vesicle is affected by their translational motion (Fig. 11a).

$\triangle$ CRITICAL STEP This transport mode is most conveniently carried out using DIC microscopy imaging.

(ii) Move the carbon fiber and the needle to opposite sides of the daughter vesicle, holding it in a pincer-like manner (Fig. 11b).

$\triangle$ CRITICAL STEP Be careful not to cleave the multilamellar reservoir off the mother vesicle in the network, because it is needed as lipid supply for the transport process.

(iii) Manipulate the needle toward the microelectrode with the fine manipulators (Fig. 11c, blue triangles). Adjust the position of the pincers so that the elastic vesicle cannot move out. Observe Marangoni flow toward the daughter

Figure 11 | Marangoni transport along a lipid nanotube in a two-vesicle network.

(a) Generation of the network by electroporation and pressure injection to a MLV-connected GUV. A small vesicle is entrapped at the tube entrance close to the mother vesicle.

(b) Carbon microelectrode and injection needle are positioned in a pincer-like arrangement around the daughter vesicle. The red arrow shows the small entrapped vesicle, which starts to move along the tube. (c) Force is exerted upon the vesicle, depicted by the blue arrowheads in the panel. The force increases lateral membrane tension in the vesicle, which is released by spontaneous Marangoni flow of membrane material from the GUV-connected MLV along the tube. The small vesicle is being carried along toward the source of tension, depicted by the position of the red arrows.

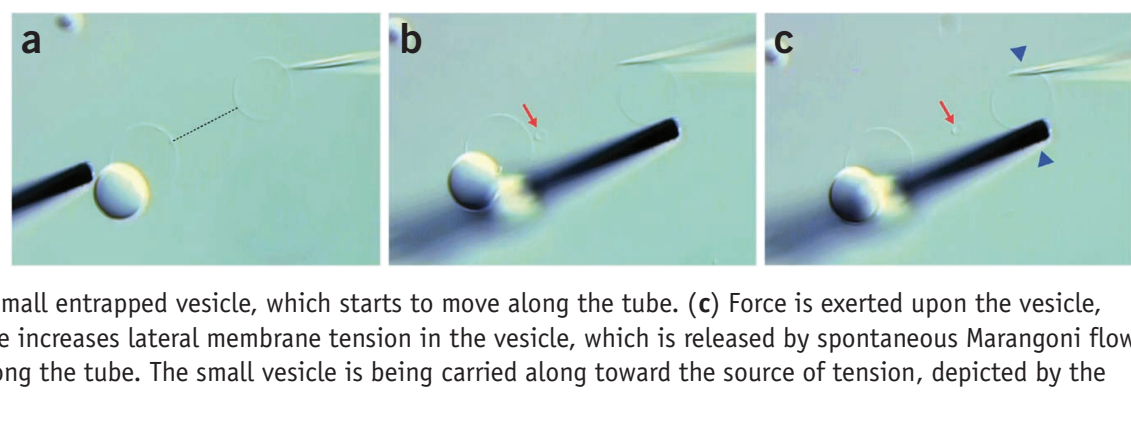


Figure 12 | Electrophoretic transport of carboxylated nanoparticles along a lipid nanotube in a two-vesicle network. (a) Bright-field DIC microscopic image of a vesicle with internalized $20 \mathrm{~nm}$ carboxylated fluorescent latex beads in between two gel-capped needles with internal $\mathrm{Ag} / \mathrm{AgCl}$ electrodes. The spot at the tip of the right needle is a small fraction of the hydrogel plug that has been ejected from the needle during membrane electroporation. (b) A nanotube entrapped aggregate of $20-\mathrm{nm}$ latex beads is transported along the tube toward the positive electrode (yellow arrow). The excitation wavelength for the fluorophore is $488 \mathrm{~nm}$. (c) Reversing the polarity reversed transport direction (yellow arrow). The electrolyte-filled gel-capped needles prevent bulk liquid flow, but allow charges to pass through the network.

vesicle on membrane-bound objects entrapped in the tube, such as particles or small unilamellar vesicles

(Fig. 11c).

(iv) Release the pincers from the daughter vesicle by means of the fine micromanipulator. Observe Marangoni flow in the opposite direction, toward the mother liposome.

(C) Electrophoretic transport in networks $\bigcirc$ TIMING 60 min

(i) Pull an injection capillary and backfill it with $15 \mu \mathrm{l}$ of filtered fluorescent nanoparticle suspension $(0.02 \%$ solid content, see REAGENT SETUP).

(ii) Ensure that both mother and daughter vesicles of the newly created two-vesicle network from Step 22 are firmly adhered to the surface.

$\Delta$ CRITICAL STEP This transport mode is most conveniently carried out using laser-induced fluorescence wide-field microscopy imaging.

(iii) Remove the needle slowly and carefully from the two-vesicle network on the cover slip surface using the fine micromanipulator, followed by the coarse one. Replace the injection needle for the one backfilled with nanoparticle solution from Step 23C(i) and move it to the surface, close to the mother vesicle.

\section{? TROUBLESHOOTING}

(iv) Microinject the nanoparticle suspension into the mother vesicle.

\section{? TROUBLESHOOTING}

(v) Remove the needle and microelectrode carefully from the droplet, using the fine and then the coarse micromanipulator.

(vi) Mount two $\mathrm{Ag} / \mathrm{AgCl}$ electrophoresis electrodes to the electrode holders.

(vii) Immerse the electrodes and bring them close to the target vesicle (Fig. 12a). ? TROUBLESHOOTING

(viii) Connect a DC power supply (minimum output: $250 \mathrm{mV}$ ) in parallel to the pulse generator.

(ix) Make sure that the DC power supply is turned off. Bring one of the electrodes in contact to the vesicle and apply a pulse of $20 \mathrm{~V}$, for $10 \mathrm{~ms}$ duration, using the pulse generator. Insert the needle into the vesicle.

(x) Bring the other electrode close to the vesicle, so that it touches the membrane. Inverse pulse polarity. Apply a pulse of $20 \mathrm{~V}$, for $10 \mathrm{~ms}$ duration, using the pulse generator. Insert the second needle into the vesicle.

(xi) Pull one electrode out of the vesicle and create a nanotube.

(xii) Turn the pulse generator off. Adjust the DC power supply to 100-250 mV. Set the positive pole to the electrode inside the vesicle, if transport of negatively charged particles from the tube into the vesicle is desired (Fig. 12b), or to the electrode at the end of the tube, if electromigration from the vesicle through the nanotube is desired (Fig. 12c). Turn on the DC power and record electromigration. Reverse polarity for a reverse of transport direction (Fig. 12c).

$\triangle$ CRITICAL STEP Single nanoparticles and polyelectrolytes, such as DNA, aggregate clusters of nanoparticles and small vesicles on the tube, are subject to electrophoretic transport through nanotubes. Injection of such materials can require larger injection needle orifices, typically $\sim 500 \mathrm{~nm}$, to avoid clogging. Electrophoresis of nanoparticles in networks can be beneficially combined with single-particle detection in a confocal microscopy setting ${ }^{35}$.

\section{? TROUBLESHOOTING}

Troubleshooting advice can be found in Table 1. 
PROTOCOL

TABLE 1 | Troubleshooting table.

\begin{tabular}{|c|c|c|c|}
\hline \multirow[t]{2}{*}{4} & $\begin{array}{l}\text { Poor vesicle } \\
\text { preparation yield }\end{array}$ & $\begin{array}{l}\text { Expired lipid stock } \\
\text { solution or aged/ } \\
\text { oxidation-damaged SPE }\end{array}$ & $\begin{array}{l}\text { Use a recently prepared stock solution that has been properly } \\
\text { stored. Phospholipids are sensitive to oxidative damage, especially } \\
\text { at room temperature. Make sure that the desiccation time is not too } \\
\text { short. Inspect the shape of the dry spot }\end{array}$ \\
\hline & & Incomplete dehydration & $\begin{array}{l}\text { If there is no bevel formed around the evaporated droplet with salt } \\
\text { crystals in the center, the drying time is too short and the vesicles } \\
\text { are not sufficiently dehydrated }\end{array}$ \\
\hline
\end{tabular}

$17,23 \mathrm{~A}(\mathrm{v}), \quad$ Needle or electrode 23C(iii), cannot be located 23C(vii) under microscope

Needle vibrates or crashes frequently into the surface

\section{$19,23 \mathrm{~A}(\mathrm{vi}), \quad$ No membrane 23C(iv) penetration with injection needle}

20, 23A(vi), Needle becomes 23C(iv) clogged
Needle tip is not in the center of the objective or needle tip is too far away from the focus

Needle is too close to the surface

Needle is mounted unstably and vibrates

Faulty or missing electrical connections to the pulse generator. Wrong pulse polarity

Clogged needle

Needle opening too large

Compensation pressure is too high

Air bubble in needle

Wrong needle size

Unwanted particles in injection solution
The needle/electrode tips have to be positioned in the center of the microscope field of view before the surface can be approached, otherwise the needle can crash into the surface and break. Before you lower the needle into the droplet, lower it to a $1 \mathrm{~mm}$ distance above the droplet surface, look from above straight down onto the objective and position the needle tip in the center of the objective lens. Lower the needle into the droplet. Adjust the focus to $>100 \mu \mathrm{m}$ above the surface. Use the coarse micromanipulator and lower the needle stepwise, $\sim 100 \mu \mathrm{m}$ at a time. After each step, move the needle repeatedly back and forth in $y$ direction (perpendicular to the needle axis) across the objective lens, until the shadow of the needle appears in the field of view. At this point, retract the needle gradually in $x$ direction (along the needle axis), until the shadow nearly disappears. Repeat lowering and translating, until the tip comes into focus. Use the fine micromanipulators to approach the surface

Whenever stage movement is required, adjust the needle position to be at least $50 \mu \mathrm{m}$ above the surface. In addition, set the distance for every operation to be carried out on the microscope, such as objective rotation, opening and closing of ports and change of filters

Make sure the micromanipulators are firmly attached to the microscope, all adjustable parts of the micromanipulators and holders are firmly fixed and the setup is placed on a vibration-dampening table (see Fig. 2)

Observe if there is movement of the membrane close to the needle when a pulse is applied. Check the connections and proper function of the pulse generator, in particular, amplitude, polarity and pulse duration

Confirm that the needle orifice is not clogged and make sure the pulse polarity is set correctly with positive potential at the needle

Make sure the needle opening is not larger than $1 \mu \mathrm{m}$

Check that in the moment of pulse application, the compensation pressure on the automatic injection pump is set to $0-1 \mathrm{hPa}$

Check for air bubbles in the needle tip and, if necessary, prepare a new injection needle

Increase the needle tip size

Make sure to filter all backfill solutions through a $0.2-\mu \mathrm{m}$ membrane filter. During an experiment, use the blow-out cleaning function of the FemtoJet pump to eject clogging particles by a high-pressure pulse 
TABLE 1 | Troubleshooting table (continued).

\begin{tabular}{|c|c|c|c|}
\hline Step & Problem & Possible reason & Possible solution \\
\hline \multirow[t]{2}{*}{22} & $\begin{array}{l}\text { Poor surface adhesion } \\
\text { and vesicle stability. } \\
\text { Surface adhesion is } \\
\text { too strong, vesicles } \\
\text { flatten on the surface. } \\
\text { Surface adhesion is }\end{array}$ & $\begin{array}{l}\text { Old, contaminated or } \\
\text { uncoated surface }\end{array}$ & $\begin{array}{l}\text { Depending on the surface used for network building, vesicle } \\
\text { adhesion differs. Use a clean, freshly produced coated surface or, } \\
\text { for short experiments, a cover slip directly out of the box. Never } \\
\text { reuse surfaces, as lipid spreading occurs, which will contaminate } \\
\text { the surface permanently }\end{array}$ \\
\hline & $\begin{array}{l}\text { too weak, vesicles will } \\
\text { not adhere }\end{array}$ & $\begin{array}{l}\text { Long experiment causes } \\
\text { droplet evaporation and } \\
\text { osmotic stress, which } \\
\text { collapses vesicles }\end{array}$ & $\begin{array}{l}\text { If experiments are prolonged over several hours, water evaporation } \\
\text { from the open droplet will change the osmotic conditions and } \\
\text { destabilize the liposomes. Pure water should be added accordingly } \\
\text { to the sample in regular intervals. A programmable syringe pump for } \\
\text { automatic dispensing is recommended }\end{array}$ \\
\hline \multirow[t]{2}{*}{$23 \mathrm{~A}($ viii) } & $\begin{array}{l}\text { Network contents } \\
\text { not visible }\end{array}$ & Photobleaching & $\begin{array}{l}\text { A common problem of fluorescent dyes is rapid photobleaching, } \\
\text { especially in wide-field laser imaging. Fluorescein-based dyes } \\
\text { bleach almost instantly and are not suitable for diffusion studies or } \\
\text { prolonged imaging }\end{array}$ \\
\hline & & $\begin{array}{l}\text { Loss of contents through } \\
\text { leaking }\end{array}$ & $\begin{array}{l}\text { Some fluorescent dyes have the tendency to leak out from vesicles, } \\
\text { in particular, when electric pulses are applied in the system, } \\
\text { which open membrane pores. If such problems occur, fluorescent } \\
\text { nanoparticles provide an alternative. Either quantum dots or } \\
\text { fluorescent latex beads are available in sizes that can be injected } \\
\text { with the current protocol (see Fig. 10) }\end{array}$ \\
\hline
\end{tabular}

\section{TIMING}

The protocol requires the preparation of a liposome suspension, which takes $\sim 18 \mathrm{~h}$ to complete. Network fabrication itself takes $\sim 4 \mathrm{~h}$ in a case in which all necessary components, such as surfaces, capillaries and reagents, are to be prepared ex novo. This time is reduced to $\sim 90$ min if these prerequisites are met beforehand. Transport experiments require between 60 and $120 \mathrm{~min}$ in addition to this time, depending on the transport mode. The procedures described here are basic procedures for two-vesicle networks. Depending on the complexity and intended purpose of the network created, the time requirements will be accordingly larger.

\section{Preparation steps}

Liposome preparation: $18 \mathrm{~h}$ (once prepared, lasts for multiple experiments)

Buffer preparation: 15 min

Membrane staining dye solution and nanoparticle suspension preparation: $30 \mathrm{~min}$

Cover slip surface treatment: 60 min (8 pieces)

Capillary needle preparation: 15 min (10 pieces)

Carbon fiber electrode fabrication: 60 min (including epoxy hardening)

Electrophoretic transport pipette preparation: 60 min (three pieces)

\section{Protocol}

Steps 1-5, de-/rehydration of lipid: 30 min

Steps 6-22, network generation: 60 min

Step 23A, diffusion-driven transport in networks: $140 \mathrm{~min}$

Step 23B, Marangoni transport in networks: $60 \mathrm{~min}$

Step 23C, electrophoretic transport in networks: $60 \mathrm{~min}$

\section{ANTICIPATED RESULTS}

The protocol presented here details the preparation of simple NVNs, consisting of two interconnected vesicles in an open buffer droplet under a microscope, as depicted in Figure $\mathbf{8 b}$. Initially, unilamellar vesicles associated with multilamellar membrane reservoirs are generated from a liposome suspension (Fig. 6a) and transferred onto a clean, surface-treated cover 
slip (Fig. 6b), wherein networks are fabricated by injection and vesicle adhesion. The networks can be extended in complexity and topology by iteration of this procedure. Figure $9 \mathrm{c}$ shows a successfully fabricated branched four-vesicle network. The protocol also describes the application of diffusion-driven (Fig. 10), membrane tension-driven (Fig. 11) and electric field-driven (Fig. 12) transport regimes, to move network constituents such as chemical compounds and nanoparticles through nanotubes.

The networks fabricated with the procedure are sufficiently stable to enable extensive experimental investigation of biomembrane and lipid nanotube properties, biochemical reactions under strong confinement conditions and singleparticle/single-molecule transport. Under ordinary laboratory conditions, the networks can be used for several hours, provided that the droplet evaporation loss is compensated by water addition.

Visualization and imaging of network-internalized solutions and the membrane itself are conveniently achieved by the use of fluorescent labels, which can be selected and introduced according to the light sources available in wide-field imaging or confocal microscopy. Figure 10 shows a confocal image of a successfully generated two-vesicle network, in which the membrane is stained with a lipophilic fluorescent label and the network interior is loaded with fluorescent nanoparticles.

Examples of experimental applications involving the generation and application of liposome-nanotube networks, as described in this protocol, in particular, initiation and control of enzymatic reactions as well as diffusion-driven substrate transport in linear and branched networks, are summarized in two recent review articles ${ }^{72,73}$.

ACKNOWLEDGMENTS We thank all previous research group members and associates, in particular, former graduate students M. Karlsson, R. Karlsson, A. Karlsson, M. Davidson, K. Sott, M. Tokarz, J. Hurtig, M. Markström, B. Bauer and T. Lobovkina, who have contributed greatly to the development of these protocols during the time they completed their individual projects. We also thank P. Dommersnes for advice and discussion. Our research was and is supported by the Royal Swedish Academy of Sciences, the Swedish Research council (VR), the Swedish Foundation for Strategic Research, the Knut and Alice Wallenberg Foundation and the European Research Council.

AUTHOR CONTRIBUTIONS A.J. performed the experimental procedures for network generation, Marangoni transport and took part in manuscript preparation. N.S. and H.Z. performed experimental procedures for diffusive transport. B.0. carried out lipid preparation. B.H. performed electrophoretic transport and took part in the manuscript preparation. 0.0. designed the original concept and took part in the manuscript preparation.

\section{COMPETING FINANCIAL INTERESTS The authors declare no competing} financial interests.

Published online at http://www.natureprotocols.com/.

Reprints and permissions information is available online at http://www.nature. com/reprints/index.html.

1. Belting, M. \& Wittrup, A. Nanotubes, exosomes, and nucleic acid-binding peptides provide novel mechanisms of intercellular communication in eukaryotic cells: implications in health and disease. J. Cell Biol. 183, 1187-1191 (2008).

2. Whitesides, G.M. The 'right' size in nanobiotechnology. Nat. Biotechnol. 21 1161-1165 (2003).

3. Davis, D.M. \& Sowinski, S. Membrane nanotubes: dynamic long-distance connections between animal cells. Nat. Rev. Mol. Cell Biol. 9, 431-436 (2008).

4. Veranic, P. et al. Different types of cell-to-cell connections mediated by nanotubular structures. Biophys. J. 95, 4416-4425 (2008).

5. Hurtig, J., Chiu, D.T. \& Onfelt, B. Intercellular nanotubes: insights from imaging studies and beyond. Wiley Interdiscip. Rev. Nanomed. Nanobiotechnol. 2, 260-276 (2010).

6. Onfelt, B. et al. Structurally distinct membrane nanotubes between human macrophages support long-distance vesicular traffic or surfing of bacteria. J. Immunol. 177, 8476-8483 (2006).

7. Gurke, S., Barroso, J.F.V. \& Gerdes, H.H. The art of cellular communication: tunneling nanotubes bridge the divide. Histochem. Cell Biol. 129, 539-550 (2008).

8. Lucas, W.J., Ham, L.K. \& Kim, J.Y. Plasmodesmata-bridging the gap between neighboring plant cells. Trends Cell Biol. 19, 495-503 (2009).

9. Lone, B. Computational nanotechnology in biomedical nanometrics and Nano-materials. J. Comput. Theor. Nanosci. 6, 2146-2151 (2009).
10. Yum, K., Wang, N. \& Yu, M.F. Nanoneedle: a multifunctional tool for biological studies in living cells. Nanoscale 2, 363-372 (2010).

11. Wong, I.Y., Almquist, B.D. \& Melosh, N.A. Dynamic actuation using nano-bio interfaces. Materials Today 13, 14-22 (2010).

12. Shvedova, A.A., Kagan, V.E. \& Fadeel, B. Close encounters of the small kind: adverse effects of man-made materials interfacing with the nanocosmos of biological systems. Annu. Rev. Pharmacol. Toxicol. 50, 63-88 (2010).

13. Fadeel, B., Kasemo, B., Malmsten, M. \& Stromme, M. Nanomedicine: reshaping clinical practice. J. Intern. Med. 267, 2-8 (2010).

14. Karlsson, A. et al. Molecular engineering-networks of nanotubes and containers. Nature 409, 150-152 (2001).

15. Karlsson, M. et al. Biomimetic nanoscale reactors and networks. Annu. Rev. Phys. Chem. 55, 613-649 (2004).

16. Karlsson, A. et al. Controlled initiation of enzymatic reactions in micrometer-sized biomimetic compartments. J. Phys. Chem. B 109, 1609-1617 (2005).

17. Bauer, B., Davidson, M. \& Orwar, 0. Direct reconstitution of plasma membrane lipids and proteins in nanotube-vesicle networks. Langmuir 22, 9329-9332 (2006).

18. Walde, P., Cosentino, K., Engel, H. \& Stano, P. Giant vesicles: preparations and applications. Chem. Bio. Chem. 11, 848-865 (2010).

19. Criado, M. \& Keller, B.U. A membrane fusion strategy for single-channel recordings of membranes usually non-accessible to patch-clamp pipette electrodes. FEBS Lett. 224, 172-176 (1987).

20. Karlsson, M. et al. Micropipet-assisted formation of microscopic networks of unilamellar lipid bilayer nanotubes and containers. Langmuir 17, 6754-6758 (2001).

21. Lasic, D.D. Liposomes: From Physics to Applications (Elsevier Science, 1993).

22. Luisi, P.L. \& Walde, P. Giant Vesicles (Wiley, 1999).

23. Barber, M.A. The pipette method in the isolation of single microorganisms and in the inoculation of substances into living cells. Phillipp. J. Sci. B. Trop. Med. 9, 307-360 (1914).

24. Taylor, C.V. An accurately controllable micropipette. Science 51, 617-618 (1920).

25. Hamill, 0.P., Marty, A., Neher, E., Sakmann, B. \& Sigworth, F.J. Improved patch-clamp techniques for high-resolution current recording from cells and cell-free membrane patches. Pflugers Arch. Eur. J. Physiol. 391, 85-100 (1981).

26. Neumann, E., Schaeferridder, M., Wang, Y. \& Hofschneider, P.H. Genetransfer into mouse lyoma cells by electroporation in high electric-fields. EMBO J. 1, 841-845 (1982).

27. Lundqvist, J.A. et al. Altering the biochemical state of individual cultured cells and organelles with ultramicroelectrodes. Proc. Natl. Acad. Sci. USA 95, 10356-10360 (1998).

28. Wang, M.Y., Orwar, 0., Olofsson, J. \& Weber, S.G. Single-cell electroporation. Anal. Bioanal. Chem. 397, 3235-3248 (2010). 
29. Karlsson, M. et al. Electroinjection of colloid particles and biopolymers into single unilamellar liposomes and cells for bioanalytical applications. Anal. Chem. 72, 5857-5862 (2000).

30. Davidson, M. et al. Fluid mixing in growing microscale vesicles conjugated by surfactant nanotubes. J. Am. Chem. Soc. 127, 1251-1257 (2005).

31. Sott, K. et al. Controlling enzymatic reactions by geometry in a biomimetic nanoscale network. Nano Lett. 6, 209-214 (2006).

32. Lizana, L. \& Konkoli, Z. Diffusive transport in networks built of containers and tubes. Phys. Rev. E 72, 026305 (2005).

33. Lizana, L., Konkoli, Z. \& Orwar, 0. Tunable filtering of chemical signals in a simple nanoscale reaction-diffusion network. J. Phys. Chem. B 111, 6214-6219 (2007).

34. Karlsson, R. et al. Moving-wall-driven flows in nanofluidic systems. Langmuir 18, 4186-4190 (2002).

35. Tokarz, M. et al. Single-file electrophoretic transport and counting of individual DNA molecules in surfactant nanotubes. Proc. Natl. Acad. Sci. USA 102, 9127-9132 (2005).

36. Tokarz, M., Hakonen, B., Dommersnes, P., Orwar, 0. \& Akerman, B. Electrophoretic transport of latex particles in lipid nanotubes. Langmuir $\mathbf{2 3}$ 7652-7658 (2007).

37. Dommersnes, P.G., Orwar, O., Brochard-Wyart, F. \& Joanny, J.F. Marangoni transport in lipid nanotubes. Europhys. Lett. 70, 271-277 (2005).

38. Lobovkina, T., Dommersnes, P., Hurtig, J., Joanny, J.F. \& Orwar, O. Zipper dynamics of surfactant nanotube junctions. Phys. Rev. Lett. 97, 188105/188101-188105/188104 (2006).

39. Evans, E. \& Rawicz, W. Entropy-driven tension and bending elasticity in condensed-fluid membranes. Phys. Rev. Lett. 64, 2094-2097 (1990).

40. Lobovkina, T., Dommersnes, P.G., Tiourine, S., Joanny, J.F. \& Orwar, 0. Shape optimization in lipid nanotube networks. Eur. Phys. J. E 26, 295-300 (2008).

41. Lizana, L., Bauer, B. \& Orwart, 0. Controlling the rates of biochemical reactions and signaling networks by shape and volume changes. Proc. Natl. Acad. Sci. USA 105, 4099-4104 (2008).

42. Lobovkina, T. et al. Mechanical tweezer action by self-tightening knots in surfactant nanotubes. Proc. Natl. Acad. Sci. USA 101, 7949-7953 (2004).

43. Karlsson, M. et al. Formation of geometrically complex lipid nanotubevesicle networks of higher order topologies. Proc. Natl. Acad. Sci. USA 99, 11573-11578 (2002).

44. Onoue, Y. et al. A giant liposome for single-molecule observation of conformational changes in membrane proteins. Biochim. Biophys. Acta Biomemb. 1788, 1332-1340 (2009).

45. Sparreboom, W., van den Berg, A. \& Eijkel, J.C.T. Principles and applications of nanofluidic transport. Nat. Nanotechnol. 4, 713-720 (2009).

46. Fernandes, P. Miniaturization in biocatalysis. Int. J. Mol. Sci. 11, 858-879 (2010).

47. Lee, L.J. Polymer nanoengineering for biomedical applications. Ann. Biomed. Eng. 34, 75-88 (2006).

48. Napoli, M., Eijkel, J.C.T. \& Pennathur, S. Nanofluidic technology for biomolecule applications: a critical review. Lab Chip 10, 957-985 (2010).

49. Sparreboom, W., van den Berg, A. \& Eijkel, J.C.T. Transport in nanofluidic systems: a review of theory and applications. New J. Phys. 12, 015004 doi:015004 10.1088/1367-2630/12/1/015004 (2010).

50. Charras, G.T. A short history of blebbing. J. Microsc. 231, 466-478 (2008)

51. Chan, Y.H.M. \& Boxer, S.G. Model membrane systems and their applications. Curr. Opin. Chem. Biol. 11, 581-587 (2007).

52. Jelinek, R. \& Silbert, L. Biomimetic approaches for studying membrane processes. Mol. BioSyst. 5, 811-818 (2009).
53. Kirby, C. \& Gregoriadis, G. Dehydration-rehydration vesicles-a simple method for high-yield drug entrapment in liposomes. Nat. Biotechnol. 2, 979-984 (1984).

54. Kikuchi, H., Yamauchi, H. \& Hirota, S. A spray-drying method for massproduction of liposomes. Chem. Pharma. Bull. 39, 1522-1527 (1991).

55. Angelova, M.I. \& Dimitrov, D.S. Liposome electroformation. Faraday Discuss. Chem. Soc. 81, 303-311 (1986).

56. Kuribayashi, K., Tresset, G., Coquet, P., Fujita, H. \& Takeuchi, S. Electroformation of giant liposomes in microfluidic channels. Measure. Sci. Technol. 17, 3121-3126 (2006).

57. Estes, D.J. \& Mayer, M. Electroformation of giant liposomes from spincoated films of lipids. Colloids Surf. B Biointerfaces 42, 115-123 (2005).

58. Estes, D.J. \& Mayer, M. Giant liposomes in physiological buffer using electroformation in a flow chamber. Biochim. Biophys. Acta Biomemb. 1712, 152-160 (2005).

59. Liu, H.Q. et al. Lipid nanotube formation from streptavidin-membrane binding. Langmuir 24, 3686-3689 (2008).

60. Roux, A. et al. A minimal system allowing tubulation with molecular motors pulling on giant liposomes. Proc. Natl. Acad. Sci. USA 99, 5394-5399 (2002).

61. Pascoal, P., Kosanic, D., Gjoni, M. \& Vogel, H. Membrane nanotubes drawn by optical tweezers transmit electrical signals between mammalian cells over long distances. Lab Chip 10, 2235-2241 (2010).

62. Mathivet, L., Cribier, S. \& Devaux, P.F. Shape change and physical properties of giant phospholipid vesicles prepared in the presence of an AC electric field. Biophys. J. 70, 1112-1121 (1996).

63. Perutkova, S., Kralj-Iglic, V., Frank, M. \& Iglic, A. Mechanical stability of membrane nanotubular protrusions influenced by attachment of flexible rod-like proteins. J. Biomech. 43, 1612-1617 (2010).

64. Davidson, M., Karlsson, M., Sinclair, J., Sott, K. \& Orwar, 0. Nanotubevesicle networks with functionalized membranes and interiors. J. Am. Chem. Soc. 125, 374-378 (2003).

65. Markstrom, M., Gunnarsson, A., Orwar, 0. \& Jesorka, A. Dynamic microcompartmentalization of giant unilamellar vesicles by sol gel transition and temperature induced shrinking/swelling of poly( $\mathrm{N}$-isopropyl acrylamide). Soft Matter 3, 587-595 (2007).

66. Karlsson, A. et al. Nanofluidic networks based on surfactant membrane technology. Anal. Chem. 75, 2529-2537 (2003).

67. Lobovkina, T. et al. Pattern formation of different geometries and topologies as well as knotted structures in lipid nanotube networks. Biophys. J. 88, 208a (2005).

68. Konings, A.W.T. Lipid peroxidation in liposomes. in Liposome Technology Vol. 1 (ed. Gregoriadis, G.) 139-161 (CRC Press, 1984).

69. Sott, K. et al. Micropipet writing technique for production of twodimensional lipid bilayer nanotube-vesicle networks on functionalized and patterned surfaces. Langmuir 19, 3904-3910 (2003).

70. Markstrom, M., Lizana, L., Orwar, 0. \& Jesorka, A. Thermoactuated diffusion control in soft matter nanofluidic devices. Langmuir 24, 5166-5171 (2008).

71. Jesorka, A., Markstrom, M. \& Orwar, 0. Controlling the internal structure of giant unilamellar vesicles by means of reversible temperature dependent sol-gel transition of internalized poly ( $N$-isopropyl acrylamide). Langmuir 21, 1230-1237 (2005).

72. Jesorka, A. \& Orwar, 0. Complex nanotube-liposome networks. in Liposomes, Part F (Methods in Enzymology) Vol. 464 309-325 (Elsevier Academic, 2009).

73. Lizana, L., Konkoli, Z., Bauer, B., Jesorka, A. \& Orwar, 0. Controlling chemistry by geometry in Nanoscale systems. Annu. Rev. Phys. Chem. 60, 449-468 (2009). 